\title{
Review Article \\ Recent Advances: The Imbalance of Cytokines in the Pathogenesis of Inflammatory Bowel Disease
}

\author{
Qingdong Guan and Jiguo Zhang \\ Institute of Pharmacology, Taishan Medical University, Tai'an, Shandong Province 271016, China \\ Correspondence should be addressed to Qingdong Guan; qdguan@gmail.com and Jiguo Zhang; jgzhang@tsmc.edu.cn
}

Received 25 October 2016; Accepted 22 February 2017; Published 21 March 2017

Academic Editor: Julio Galvez

Copyright (c) 2017 Qingdong Guan and Jiguo Zhang. This is an open access article distributed under the Creative Commons Attribution License, which permits unrestricted use, distribution, and reproduction in any medium, provided the original work is properly cited.

Cytokines play an important role in the immunopathogenesis of inflammatory bowel disease (IBD), including Crohn's disease and ulcerative colitis, where they drive and regulate multiple aspects of intestinal inflammation. The imbalance between proinflammatory and anti-inflammatory cytokines that occurs in IBD results in disease progression and tissue damage and limits the resolution of inflammation. Targeting cytokines have been novel strategies in the treatment of IBD. Recent studies show the beneficial effects of anticytokine treatments to IBD patients, and multiple novel cytokines are found to be involved in the pathogenesis of IBD. In this review, we will discuss the recent advances of novel biologics in clinics and clinical trials, and novel proinflammatory and anti-inflammatory cytokines found in IBD with focusing on IL-12 family and IL-1 family members as well as their relevance to the potential therapy of IBD.

\section{Introduction}

Inflammatory bowel disease (IBD) is a chronic inflammatory disease of the gastrointestinal tract, which clinically contains Crohn's disease (CD), ulcerative colitis (UC), and other conditions $[1,2]$. The inflammation of the intestinal mucosa in IBD is characterized by episodes of abdominal pain, diarrhea, bloody stools, weight loss, and the influx of neutrophils, macrophages, and other immune cells that produce cytokines, proteolytic enzymes, and free radicals that result in inflammation and ulceration $[1,3]$.

IBD is a lifelong disease occurring early in life in both males and females. The incidence and prevalence of IBD markedly increased over the second half of the twentieth century, and since the beginning of the twenty-first century, IBD has been considered one of the most prevalent gastrointestinal diseases [4-7]. Estimates indicate that as of 2005, about 1.4 million Americans and several millions persons worldwide have been diagnosed with IBD. Roughly $30 \%$ are children and adults between 10 and 30 years of age [8]. The incidence of $\mathrm{CD}$ in North America has been estimated at between 3.1 and 14.6 per 100,000 , with a prevalence of between 26.0 and 198.5 per 100,000 [1]. For UC, both incidence and prevalence are estimated at between 2.2 and 14.3 and 37.5 and 229 per 100,000, respectively [1].

Although the cause of IBD remains unknown, considerable progress has been made in the recent years to unravel the pathogenesis of this disease. Studies have provided evidence that the pathogenesis of IBD is associated with genetic susceptibility of the host, intestinal microbiota, other environmental factors, and immunological abnormalities [9-11]. The immunological dysregulation in IBD is characterized by epithelial damage (abnormal mucus production, defective repair); expansion of inflammation driven by intestinal flora and a large number of cells infiltrating into the lamina propria including T cells, B cells, macrophages, dendritic cells (DC), and neutrophils; and a failure of immune regulation to control the inflammatory response $[2,4,12]$. A large number of soluble mediators are actively secreted by the activated lamina propria cells in the local tissue, including proinflammatory cytokines (TNF, IFN- $\gamma$, IL-6, IL-12, IL-21, IL-23, IL-17, integrin, etc.) and anti-inflammatory cytokines (IL-10, TGF $\beta$, 
IL-35, etc.) $[2,11]$. CD is usually designated as a Th1 and Th17 condition with elevated production of IL-12, IL-23, IFN- $\gamma$, and IL-17, whereas UC is usually characterized as a Th2 and Th9 condition with increased production of IL-13, IL-5, and IL-9 [2]. The roles of cytokines in initiating, mediating, perpetuating, and controlling intestinal inflammation and tissue injury have been intensely studied because they are the key players in the pathogenesis of IBD and they may be the potential therapeutic targets $[11,13]$. Antibodies against TNF, IL-12/IL-23p40, IFN- $\gamma$, IL-6R, IL-11, IL-13, IL-17A, integrin, and recombinant IL-10 and IFN$\beta$ have been tested or applied in clinics to treat IBD patients [11]. This review will describe recent advances in biologics treatment or clinical trials for IBD patients and novel cytokines found in the pathogenesis of IBD with focusing on IL-12 family and IL-1 family members.

\section{Recent Advances in Biologics Treatment and Clinical Trials in IBD}

Monoclonal antibodies against TNF are the first biologics approved and widely used for the treatment of both CD and UC, including infliximab, adalimumab, and certolizumab pegol, which have demonstrated good clinical efficacy in their abilities to induce remission and maintain steroidfree remission $[14,15]$. However, around $20 \%$ of patients do not respond to anti-TNF, and over 30\% eventually lose response [16]. Moreover, these antibody treatments can increase the risk of infections and malignancies [16]. Therefore, other new biologics are currently being developed for both anti-TNF-naive and TNF-resistant IBD patients [16]. In 2014, monoclonal antibody against integrin $\alpha 4 \beta 7$, vedolizumab, was approved to treat adults with moderate-to-severe active CD or UC. Vedolizumab also benefits one-third of patients with IBD who failed to previous anti-TNF therapy in a phase III trial [17]. Vedolizumab is efficacious and safe in pediatric IBD patients too, with UC patients experiencing earlier and higher rates of remission than $\mathrm{CD}$ patients in a multicenter experience trial [18]. Mongersen, an oral SMAD7 antisense oligonucleotide, which targets SMAD7 to increase the activity of TGF $\beta$, induces significantly higher rates of remission and clinical response than placebo in patients with active $\mathrm{CD}$ in a phase II trial [19]. Tofacitinib is an oral inhibitor of JAK1, 2, and 3 to block signaling pathways of $\gamma$ chain-containing cytokines, including IL-2, IL-4, IL-7, IL-9, IL-15, and IL21 [16]. In a phase II trial, tofacitinib induces clinical responses and remission in patients with moderate-tosevere UC, not for CD patients [20]. Add-on therapy with tralokinumab targeting IL-13 does not significantly improve clinical response but induces a higher clinical remission rate than placebo in a phase IIa trial, suggesting that tralokinumab may benefit some patients with UC [21]. But not all these biological agents achieve clinical responses. Another monoclonal antibody against IL-13, anrukinzumab, does not induce clinical benefit for patients with active UC in a phase IIa trial [22]. Several other biologics are under the clinical evaluation, such as anti-IL-12/IL-23p40.

\section{IL-12 Family}

IL-12 family of cytokines contains four cytokines, IL-12, IL-23, IL-27, and IL-35. Each member is composed of a helical $\alpha$ subunit (p35, p19, and p28) and a $\beta$-subunit (p40 and EBI3) structurally similar to the extracellular domain of type 1 cytokine receptors $[23,24]$. IL-12 is composed of p35 and p40, IL-23 is composed of p19 and p40, IL-27 is composed of p28 and EBI3, and IL-35 contains p35 and EBI3 [23, 24]. The binding of the IL-12 family of cytokines to their corresponding receptors will activate JAK/STAT signaling pathways, leading to transcription of target genes that mediate biological activities. The IL-12 family of cytokines has emerged as important regulators of host immunity [23, 24].

IL-12 is predominantly produced by DC, monocytes, and macrophages following recognition of pathogenic structures by toll-like receptors and other receptors $[25,26]$. IL-12 induces the production of IFN- $\gamma$, favours the differentiation of Th1, and forms a link between innate and adaptive immunity [27]. IL-23 is also predominantly produced by activated dendritic and phagocytic cells [26, 28, 29]. IL-23 plays an important role in stabilizing/amplifying Th17 proliferation [30, 31].

Studies have demonstrated that IL-12 and IL-23 play important roles in the pathogenesis of CD. Multiple studies indicate that IL-12 is overproduced in the gastric mucosa, lamina propria mononuclear cells, and macrophages in CD, and macrophages isolated from the inflammatory lesions of patients with CD produce increased amounts of IL-12 ex vivo [32-35]. Recent studies have highlighted the roles of IL-23 in the pathogenesis of CD [4, 36-38]. The colonic level of IL-23 is increased in patients with CD [39]. The myeloid DC from the mesenteric lymph nodes of CD patients secretes high levels of IL-23 [40]. The expression of IL-23R is upregulated in the lamina propria isolated from CD [41]. And the upregulated expression of IL-23R is correlated with IFN- $\gamma$ [41]. Recently, a genome-wide association study has identified numerous SNP in $I L-23 R$, with high association for CD and UC $[42,43]$. Of interest, the G149R, V362I and R381Q IL-23R $\alpha$ chain variants, confers the protective effects in patients with CD and UC [43, 44]. These protective effects are due to impaired protein stability and intracellular trafficking, then leading to decrease the surface receptor expression and further reduce STAT signaling pathway [44]. Yen et al. used IL-10 knockout mice, a spontaneous IBD model, and showed that the development of colitis was suppressed by IL-23p19 deficiency but not IL-12p35 deficiency in IL- $10^{-/-}$mice; administration of IL-23 accelerated the onset of colitis and promoted inflammation through IL-17- and IL-6-dependent mechanisms [36]. As IL-12 and IL-23 share p40 subunit, targeting IL-12/IL-23p40 has been widely tested to improve intestinal inflammation in preclinical studies and clinical trials. Briakinumab and ustekinumab are human monoclonal antibodies against IL-12/IL-23p40 which induced clinical response and remission in a certain subtype of patients with CD [45-48]. In a phase IIb trial, briakinumab induced numerically greater rates of remission and response in moderate-to-severe active CD patients when compared to 
placebo treatment [47]. In a phase III-randomized trial, ustekinumab treatment induced significant clinical response and remission in moderate-to-severe CD patients refractory to prior TNF antagonists [48]. Since CD is a chronic inflammatory disease, it usually needs long-term treatment while monoclonal antibodies have short half-life. To overcome these disadvantages, we developed an IL-12/IL-23p40 peptide-based vaccine which induced relative long-lasting antibodies against IL-12/IL-23, and immunization with this vaccine improved TNBS-induced acute and chronic murine colitis by downregulating IL-12, IL-23, TNF, and IFN- $\gamma$ and reducing fibrosis $[49,50]$. Interestingly, immunization with the same vaccine can also ameliorate allergic murine skin and airway inflammation [51].

IL-27 is predominately produced by DC, macrophages and monocytes following stimulation by different immune stimuli $[52,53]$. IL-27 regulates both innate and adaptive immune responses, including activating innate immune cells (e.g., macrophages), promoting Th1 and type 1 regulatory $\mathrm{T}$ cell differentiation, and inhibiting the differentiation of Th2, Th17, and Treg cells [52, 53]. However, under certain conditions, the regulatory function of IL-27 can be deviated, such as inhibiting Th1 but promoting Treg development [52, 53].

The important role of IL-27 in the pathogenesis of IBD has been indicated. Multiple studies have implicated IL-27 gene polymorphism and mutation are associated with the risk of IBD [54]. IL-27 gene expression level is increased in the local colon tissue of patients with active UC or active CD [55]. The proinflammatory and anti-inflammatory effects of IL-27 have been observed in IBD [54]. Oral delivery of recombinant IL-27 food-grade bacterium Lactococcus lactis ameliorates the murine colitis in a $\mathrm{T}$ cell-dependent colitis model, including improving survival, decreasing clinical score and pathologic score, downregulating inflammatory cytokines, and increasing IL-10 [56]. Subcutaneous administration with IL-27 also attenuates TNBS-induced intestinal inflammation with reducing pathologic score, decreasing inflammatory cytokines, and inhibiting Th17 cells [57]. IL-27R $\mathrm{R}^{-/-}$mice exhibited earlier onset and significantly increased severity of intestinal inflammation when compared to wild-type controls after DSS treatment [58]. These studies demonstrated the protective role of IL-27 in IBD.

On the other hand, some studies indicated the proinflammatory roles of IL-27 in IBD based on blocking IL-27 receptor signaling pathway [54]. IL-27R $\alpha^{-/-}$mice developed less severe intestinal inflammation after DSS treatment when compared to wild-type mice, characterized by reducing inflammatory cytokines (IL-6, TNF, and IFN- $\gamma$ ). Another study showed that IL-27R $\alpha^{-/-}$effector T cells had poor proliferation and less IFN- $\gamma$ secretion; transfer of IL-27R $\alpha^{-1-} \mathrm{T}$ cells results in diminished weight loss and reduced intestinal inflammation when compared to transferring of wild-type $\mathrm{T}$ cells [59]. Visperas et al. showed that IL-27R $\alpha^{-1-} \mathrm{TCR} \beta^{-1-}$ recipients did not develop intestinal inflammation after naive $\mathrm{CD} 4^{+} \mathrm{T}$ cell infusion because these recipient mice had poor Th17 differentiation and lower expression of IL- 6 and IL$1 \beta$ by antigen-presenting cells; while IL- $27 \mathrm{R} \alpha^{+/+} \mathrm{TCR} \beta^{-/-}$ recipients developed severe colitis after naive $\mathrm{CD} 4^{+} \mathrm{T}$ cell infusion [60]. These results indicate the complicated roles of IL-27 in IBD, which requires further investigation.

$I L-35$ is a newly identified cytokine of the IL-12 family, which is mainly produced by Treg, activated B cells, and DC $[11,23,61]$. IL-35 can induce naive human and mouse $\mathrm{T}$ cells to differentiate into regulatory $\mathrm{T}$ cells (iTr35 cells), which do not express Foxp3, IL-10, and TGF but suppress $\mathrm{T}$ cell responses through IL-35 [23]. IL-35 suppresses T cell proliferation by inducing cell cycle arrest in G1 phase without inducing apoptosis [23]. In addition, IL-35 promotes the differentiation of human $\mathrm{B}$ cells into Breg which produces IL-35 and IL-10, and IL-35-producing B cells play an important role in the suppressive regulation of immunity [62]. Multiple studies have demonstrated the important regulatory role of IL-35 in IBD. Recently, two groups showed that IL-35 levels are significantly decreased in the serum but increased in the local colon tissue, and high IL-35 secreting intestinal Breg and circulating regulatory $\mathrm{CD} 4^{+} \mathrm{T}$ and $\mathrm{CD} 8^{+} \mathrm{T}$ cells are found in patients with active IBD $[63,64]$; the level of IL-35 in the serum is inversely correlated with that of the activity of UC [64]. In T cell-dependent murine colitis model, $\mathrm{EBI}^{-/-}$mice (lacking both IL-27 and IL-35) develop early onset and severe colitis with shorten survival time, when compared to IL-27p $28^{-/-}$mice (lacking IL-27 only) [65], while recombinant IL-35 treatment significantly limited the development of several forms of experimental colitis and decreased levels of markers of Th1 and Th17 cells [65]. Adoptive transfer of IL35-induced iTr35 can ameliorate the development of murine colitis [23]. These results suggest IL-35 may be an important regulator of IBD, with the potential for the treatment of IBD.

\section{IL-1 Family}

IL-1 family includes seven agonistic cytokines (IL- $1 \alpha$, IL- $1 \beta$, IL-18, IL-33, IL-36 $\alpha$, IL-36 $\beta$, and IL-36 $\gamma$ ), three receptor antagonists (IL-1R $\alpha$, IL-36R $\alpha$, and IL-38), and one antiinflammatory cytokine (IL-37) [66]. The IL-1 receptor family members include 10 molecules, from IL-1R1 to IL-1R10 [66]. Upon cytokine binding, IL-1 receptors heterodimerize, which recruit intracellular signaling molecules, including MyD88, IRAK, and TRAF6, then activate NF- $\kappa$ B, p38, JNK, and/or MAPK transcription factors, leading to transcription of target genes (such as IL-6, IL-5, IL-4, IL-8, MCP-1, and COX-2) [67]. All innate immune cells express and/ or are affected by the IL- 1 family members. Moreover, the IL-1 family members play an important role in the differentiation and function of polarized innate and adaptive lymphoid cells [66]. Here, we will focus on the review of the roles of novel cytokines IL-33, IL-36, and IL-37 in the pathogenesis of IBD.

IL-33 is widely expressed by many cell types, such as epithelial cells, fibroblasts, smooth muscle cells, and endothelial cells $[66,68]$. IL-33 exerts its biological function through binding of its receptor T1/ST2 [69]. Many immune cells are responsive to IL-33 and express T1/ST2 on their surface, and the main effects of IL-33 are involve in inflammation and type 2 immunity, including activation and accumulation of type 2 innate lymphoid cells, Th2, and M2 polarized macrophages $[66,68]$. It has shown that IL-33 plays a role in the 
pathogenesis of many diseases, such as infection and autoimmune diseases [69].

IL-33 is constitutively expressed in epithelial cells at barrier sites of the gut, which can be regulated by epidermal growth factor [70]. In response to tissue damage, IL-33 functions as an alarmin by driving Th2, Th17, and Treg responses and influencing wound healing of damaged tissue $[68,71]$. Through screening over 1500 IBD patients, a recent study shows that IL-33 polymorphisms contribute to the risk of IBD [72]. IL-33, along with ST2, is significantly increased in the inflamed IBD biopsy samples, especially in UC [72-74]. Furthermore, the expression of IL-33 is correlated with the inflammatory status [69]. At the disease remission stage of UC after anti-TNF treatment, IL-33 loses its expression in colonic crypts [75]. Soluble ST2 is a sensitive marker of treatment response and clinical outcome of UC [76]. IL-33 is also found to be increased in the colon tissue of animal colitis models [77, 78]. Deficiency of ST2 protects mice from TNBS- and DSS-induced murine colitis [79]. Administration of recombinant IL-33 exaggerates the severity of DSS-induced acute colitis, which is associated with marked elevation of IL-4, IL-5, and IL-13, significant reduction of IL-17 and IFN- $\gamma$ in the colon tissue, impairment of the epithelial barrier, and delay of wound healing of the injured colonic epithelia [79-81]. But another group shows that administration of IL-33 protects DSS-induced acute colitis through inducing group 2 innate lymphoid cells with the expression of IL-4 and IL-5 and growth factor amphiregulin [78]. In TNBS-induced acute colitis, IL-33 administration protects the intestinal inflammation by promoting Th2/Foxp ${ }^{+}$Treg cells [77]. In DSS-induced chronic colitis, IL-33 ameliorates the intestinal inflammation through suppressing Th1 and Th17 responses [82]. However, in SAMP1/YitFc spontaneous chronic murine colitis model, IL-33 administration worsens the chronic intestinal inflammation by enhancing eosinophil infiltration and increasing pathogenic Th2 response [83]; these effects can be reversed by blockade of IL-33 signaling or depletion of eosinophils and required gut microbiomes $[83,84]$. These animal findings indicate that IL-33 may confer protection from injury or lead to inflammation although the behind mechanisms remain largely undefined.

IL-36 contains three agonistic ligands (IL-36 $\alpha$, IL-36 $\beta$, and IL-36 $\gamma$ ) and one antagonist ligand (IL-36Ra) [85], which bind to the same heterodimeric receptor. Not like other members of the IL-1 family, IL-36 is mainly expressed in keratinocytes, bronchial epithelia, brain tissues, and monocytes/ macrophages [85]. IL-17 and TNF can induce the expression of IL-36 in keratinocytes, and IL-22 can synergize these effects [86]. IL-36 receptor signaling activates DC and plays a role in polarizing helper $\mathrm{T}$ cell responses [85]. IL-36 induces the secretion of proinflammatory cytokines (IL-12, IL-6, IL-1 $\beta$, and TNF) from bone marrow-derived DC, IL-4, IL-17, and IFN- $\gamma$ from CD $4^{+}$T cells [87]. IL-36 $\beta$ induces the secretion of IL-12 and IL-18 from human monocyte-derived DC, which subsequently leads to the proliferation of IFN$\gamma^{+} \mathrm{T}$ lymphocytes [88]. Studies have shown that IL-36 plays a role in the pathogenesis of asthma, autoimmune diseases, psoriasis, and other diseases [85].
The mRNA expression of IL-36 $\alpha$ and IL-36 $\gamma$, not IL-36 $\beta$, is increased in the inflamed mucosa of IBD patients, especially in UC and in DSS-induced murine colitis [89, 90]. Further, it finds that $\mathrm{T}$ cells, monocytes, and plasma cells in inflamed mucosa of IBD patients are the source of IL-36 $\alpha$ and IL-36 $\gamma$ [89]. IL-36 $\alpha$ and IL-36 $\gamma$ induce the expression of CXC chemokines on human intestinal epithelial cell line HT-29 cells in dose-dependent and time-dependent manners [89]. IL-36 $\mathrm{R}^{-1-}$ mice reduced the intestinal inflammation in DSS-induced acute colitis, associated with decreased innate inflammatory cell infiltration into the colon lamina propria [91]. Similarly, after infection with the enteropathogenic bacteria Citrobacter rodentium, IL-36 $\mathrm{R}^{-/-}$mice reduced innate inflammatory cell infiltration and increased bacterial colonization in the colon, with enhanced Th17 and reduced Th1 responses [91]. Another group shows that IL-36 signaling may be important in the resolution of intestinal damage [92]. After DSS treatment, IL-36R-deficient mice reduce intestinal inflammation but significantly delay the wound healing of colonic mucosa, which is associated with the reduction of neutrophil infiltration into the colonic mucosa and reduction of barrier-protective cytokine IL22 in the colon [92]. Administration of an aryl hydrocarbon receptor agonist restores IL-22 expression and promotes full recovery from DSS treatment in IL-36R-deficient mice $[84,92]$. These studies indicate that IL-36 signaling plays a role in the pathogenesis of IBD and post injury healing, with the potential to be the target for the treatment of IBD.

$I L-37$ is expressed in diverse human tissues, such as skin, tonsil, placenta, breast, and melanoma [93-95]. IL-37 is induced in DC and peripheral blood mononuclear cells stimulated by TNF, IFN- $\gamma$, IL- $1 \beta$, and several toll-like receptor agonists [96]. The binding of IL-37 to IL-18R $\alpha / \mathrm{IL}-1 \mathrm{R} 8$ controls the regulators of cellular adhesion and migration such as FAK, Pyk2, and transcription factors such as NF-kB and MAPK to display the anti-inflammatory activities mediated via Smad3 and caspase-1 [93, 94]. Increased levels of IL-37 have been reported in multiple diseases by measuring IL-37 mRNA or protein in cells derived from patients or in serum, such as rheumatoid arthritis, melanoma, atopic dermatitis, and ankylosing spondylitis [94]. In some other diseases, IL-37 levels are found to be decreased, such as psoriasis, asthma, and allergic rhinitis [94].

There are several reports that evaluated the levels of IL-37 in IBD patients. The percentage of IL-37-secreting cells is higher in the inflamed intestine of active CD patients than that in active ulcerative and noninflamed control tissues [63]. Levels of IL-37 in the sera are increased in active IBD patients, which are conspicuously produced by circulating B cells, active natural killer cells, and monocytes $[63,64]$. In pediatric IBD patients, IL-37 protein expression is increased in submucosal lymphoid cells and correlated with histological severity score of intestinal inflammation; another IL-1 family member, IL-18, shows the similar pattern as IL-37 in these patients [97]. In vitro experiment shows that IL-37b inhibits the TNF $\alpha$ induced chemokine IP-10 expression in human colonic subepithelial myofibroblasts [98]. Animal studies demonstrate anti-inflammatory roles of IL-37 in colitis. Transgenic mice overexpressing human IL-37b (IL-37b-tg) exhibits 
reduction of DC activation and proinflammatory cytokine secretion after LPS stimulation. IL-37b-tg mice protect from DSS-induced colitis, characterized by decreasing clinical disease score, histological score, and TNF $\alpha$ and IL- $1 \beta$ production, but increasing IL-10 production in colon tissue [99]. If overexpressing IL-37b on mesenchymal stromal cells, it will greatly increase the therapeutic efficacy of mesenchymal stromal cells in DSS-induced murine colitis [100]. These results indicate that IL-37 may be useful for the treatment of IBD.

\section{Conclusion and Prospective}

Cytokines play a crucial role in driving, perpetuating, resolving, and wound healing of intestinal inflammation in IBD. Novel biologics that targeting cytokines or cytokine signaling pathway cascades are being used in clinics or being tested in clinical trials. However, these biologics only seem to have beneficial clinical effects in certain subgroups of IBD patients. This may reflect the complex of cytokine networks in the inflamed colon tissue, which are subject to types of inflammation, location, microbiota, genetic, immune cell plasticity, and others [11]. Blockade of a single cytokine in IBD patients may drive other proinflammatory cytokine pathways. Therefore, to optimize the clinical response and remission rate in IBD patients, it may require using multiple cytokine inhibitors that simultaneously block several cytokines or common cytokine signaling pathway - the JAK-STAT pathway. Taken together, anti-TNF and anti-integrin $\alpha 4 \beta 7$ have been the mainstay of biological therapy in IBD. New cytokine targets (e.g., IL-12/IL-23p40 and SMAD7), novel anti-inflammatory cytokines (e.g., IL-35 and IL-37), and personalized medicines may provide potential treatment for IBD patients.

\section{Abbreviations}

CD: Crohn's disease

DC: Dendritic cells

DSS: Dextran sulfate sodium

IBD: Inflammatory bowel disease

IFN: Interferon

IL: Interleukin

TNBS: 2,4,6-Trinitrobenzenesulfonic acid

TNF: Tumor necrosis factor

UC: Ulcerative colitis.

\section{Conflicts of Interest}

The authors declare that there is no conflict of interest regarding the publication of this paper.

\section{Authors' Contributions}

Qingdong Guan proposed the idea and drafted the manuscript. Jiguo Zhang extensively reviewed and edited the manuscript. Qingdong Guan and Jiguo Zhang designed the outline of the manuscript and had final approval of the version submitted for publication.

\section{Acknowledgments}

This work is supported by the Shandong Provincial Natural Science Foundation, China (no. ZR2011HM044), to Dr. Jiguo Zhang.

\section{References}

[1] E. Szigethy, L. McLafferty, and A. Goyal, "Inflammatory bowel disease," Child and Adolescent Psychiatric Clinics of North America, vol. 19, no. 2, pp. 301-318, 2010, ix.

[2] H. S. de Souza and C. Fiocchi, "Immunopathogenesis of IBD: current state of the art," Nature Reviews Gastroenterology \& Hepatology, vol. 13, no. 1, pp. 13-27, 2016.

[3] P. C. Stokkers and D. W. Hommes, "New cytokine therapeutics for inflammatory bowel disease," Cytokine, vol. 28, no. 4-5, pp. 167-173, 2004.

[4] J. R. Korzenik and D. K. Podolsky, "Evolving knowledge and therapy of inflammatory bowel disease," Nature Reviews Drug Discovery, vol. 5, no. 3, pp. 197-209, 2006.

[5] E. V. Loftus Jr., "Clinical epidemiology of inflammatory bowel disease: incidence, prevalence, and environmental influences," Gastroenterology, vol. 126, no. 6, pp. 15041517, 2004.

[6] V. Binder, "Epidemiology of IBD during the twentieth century: an integrated view," Best Practice \& Research Clinical Gastroenterology, vol. 18, no. 3, pp. 463-479, 2004.

[7] S. B. Hanauer, "Inflammatory bowel disease: epidemiology, pathogenesis, and therapeutic opportunities," Inflammatory Bowel Diseases, vol. 12, Supplementary 1, pp. S3-S9, 2006.

[8] G. Bamias, M. R. Nyce, S. A. De La Rue, and F. Cominelli, "New concepts in the pathophysiology of inflammatory bowel disease," Annals of Internal Medicine, vol. 143, no. 12, pp. 895-904, 2005.

[9] A. Kaser, S. Zeissig, and R. S. Blumberg, "Inflammatory bowel disease," Annual Review of Immunology, vol. 28, no. 1, pp. 573-621, 2010.

[10] I. Loddo and C. Romano, "Inflammatory bowel disease: genetics, epigenetics, and pathogenesis," Frontiers in Immunology, vol. 6, p. 551, 2015.

[11] M. F. Neurath, "Cytokines in inflammatory bowel disease," Nature Reviews Immunology, vol. 14, no. 5, pp. 329-342, 2014.

[12] M. N. Ince and D. E. Elliott, "Immunologic and molecular mechanisms in inflammatory bowel disease," The Surgical Clinics of North America, vol. 87, no. 3, pp. 681-696, 2007.

[13] M. L. Chen and M. S. Sundrud, "Cytokine networks and T-cell subsets in inflammatory bowel diseases," Inflammatory Bowel Diseases, vol. 22, no. 5, pp. 1157-1167, 2016.

[14] M. Berns and D. W. Hommes, "Anti-TNF-alpha therapies for the treatment of Crohn's disease: the past, present and future," Expert Opinion on Investigational Drugs, vol. 25, no. 2, pp. 129-143, 2016.

[15] G. W. Dryden Jr., "Overview of biologic therapy for Crohn's disease," Expert Opinion on Biological Therapy, vol. 9, no. 8, pp. 967-974, 2009.

[16] B. Ungar and U. Kopylov, "Advances in the development of new biologics in inflammatory bowel disease," Annals of Gastroenterology, vol. 29, no. 3, pp. 243-248, 2016.

[17] A. Amiot, J. C. Grimaud, L. Peyrin-Biroulet et al., "Effectiveness and safety of vedolizumab induction therapy for patients 
with inflammatory bowel disease," Clinical Gastroenterology and Hepatology, vol. 14, no. 11, pp. 1593-1601, 2016, e2.

[18] N. Singh, S. Rabizadeh, J. Jossen et al., "Multi-center experience of vedolizumab effectiveness in pediatric inflammatory bowel disease," Inflammatory Bowel Diseases, vol. 22, no. 9, pp. 2121-2126, 2016.

[19] G. Monteleone, M. F. Neurath, S. Ardizzone et al., "Mongersen, an oral SMAD7 antisense oligonucleotide, and Crohn's disease," The New England Journal of Medicine, vol. 372, no. 12, pp. 1104-1113, 2015.

[20] W. J. Sandborn, S. Ghosh, J. Panes et al., "Tofacitinib, an oral Janus kinase inhibitor, in active ulcerative colitis," The New England Journal of Medicine, vol. 367, no. 7, pp. 616-624, 2012.

[21] S. Danese, J. Rudzinski, W. Brandt et al., "Tralokinumab for moderate-to-severe UC: a randomised, double-blind, placebo-controlled, phase IIa study," Gut, vol. 64, no. 2, pp. 243-249, 2015.

[22] W. Reinisch, J. Panes, S. Khurana et al., "Anrukinzumab, an anti-interleukin 13 monoclonal antibody, in active UC: efficacy and safety from a phase IIa randomised multicentre study," Gut, vol. 64, no. 6, pp. 894-900, 2015.

[23] D. A. Vignali and V. K. Kuchroo, "IL-12 family cytokines: immunological playmakers," Nature Immunology, vol. 13, no. 8, pp. 722-728, 2012.

[24] L. Sun, C. He, L. Nair, J. Yeung, and C. E. Egwuagu, "Interleukin 12 (IL-12) family cytokines: role in immune pathogenesis and treatment of CNS autoimmune disease," Cytokine, vol. 75, no. 2, pp. 249-255, 2015.

[25] M. K. Gately, L. M. Renzetti, J. Magram et al., "The interleukin-12/interleukin-12-receptor system: role in normal and pathologic immune responses," Annual Review of Immunology, vol. 16, no. 1, pp. 495-521, 1998.

[26] C. L. Langrish, B. S. McKenzie, N. J. Wilson, M. R. de Waal, R. A. Kastelein, and D. J. Cua, "IL-12 and IL-23: master regulators of innate and adaptive immunity," Immunological Reviews, vol. 202, no. 1, pp. 96-105, 2004.

[27] G. Trinchieri, "Interleukin-12 and the regulation of innate resistance and adaptive immunity," Nature Reviews Immunology, vol. 3, no. 2, pp. 133-146, 2003.

[28] C. A. Hunter, "New IL-12-family members: IL-23 and IL-27, cytokines with divergent functions," Nature Reviews Immunology, vol. 5, no. 7, pp. 521-531, 2005.

[29] M. M. D'Elios, G. Del Prete, and A. Amedei, “Targeting IL-23 in human diseases," Expert Opinion on Therapeutic Targets, vol. 14, no. 7, pp. 759-774, 2010.

[30] K. Hirahara, K. Ghoreschi, A. Laurence, X. P. Yang, Y. Kanno, and J. J. O'Shea, "Signal transduction pathways and transcriptional regulation in Th17 cell differentiation," Cytokine \& Growth Factor Reviews, vol. 21, no. 6, pp. 425-434, 2010.

[31] I. I. Ivanov, L. Zhou, and D. R. Littman, "Transcriptional regulation of Th17 cell differentiation," Seminars in Immunology, vol. 19, no. 6, pp. 409-417, 2007.

[32] P. Parronchi, P. Romagnani, F. Annunziato et al., "Type 1 T-helper cell predominance and interleukin-12 expression in the gut of patients with Crohn's disease," The American Journal of Pathology, vol. 150, no. 3, pp. 823-832, 1997.

[33] I. Peluso, F. Pallone, and G. Monteleone, "Interleukin-12 and Th1 immune response in Crohn's disease: pathogenetic relevance and therapeutic implication," World Journal of Gastroenterology, vol. 12, no. 35, pp. 5606-5610, 2006.
[34] G. Monteleone, L. Biancone, R. Marasco et al., "Interleukin 12 is expressed and actively released by Crohn's disease intestinal lamina propria mononuclear cells," Gastroenterology, vol. 112, no. 4, pp. 1169-1178, 1997.

[35] D. Berrebi, M. Besnard, G. Fromont-Hankard et al., "Interleukin-12 expression is focally enhanced in the gastric mucosa of pediatric patients with Crohn's disease," The American Journal of Pathology, vol. 152, no. 3, pp. 667-672, 1998.

[36] D. Yen, J. Cheung, H. Scheerens et al., "IL-23 is essential for T cell-mediated colitis and promotes inflammation via IL-17 and IL-6," The Journal of Clinical Investigation, vol. 116, no. 5, pp. 1310-1316, 2006.

[37] S. Hue, P. Ahern, S. Buonocore et al., "Interleukin-23 drives innate and T cell-mediated intestinal inflammation," The Journal of Experimental Medicine, vol. 203, no. 11, pp. 2473-2483, 2006.

[38] M. C. Kullberg, D. Jankovic, C. G. Feng et al., "IL-23 plays a key role in Helicobacter hepaticus-induced T cell-dependent colitis," The Journal of Experimental Medicine, vol. 203, no. 11, pp. 2485-2494, 2006.

[39] C. Schmidt, T. Giese, B. Ludwig et al., "Expression of interleukin-12-related cytokine transcripts in inflammatory bowel disease: elevated interleukin-23p19 and interleukin27 p28 in Crohn's disease but not in ulcerative colitis," Inflammatory Bowel Diseases, vol. 11, no. 1, pp. 16-23, 2005.

[40] A. Sakuraba, T. Sato, N. Kamada, M. Kitazume, A. Sugita, and T. Hibi, "Th1/Th17 immune response is induced by mesenteric lymph node dendritic cells in Crohn's disease," Gastroenterology, vol. 137, no. 5, pp. 1736-1745, 2009.

[41] T. Kobayashi, S. Okamoto, T. Hisamatsu et al., "IL23 differentially regulates the Th1/Th17 balance in ulcerative colitis and Crohn's disease," Gut, vol. 57, no. 12, pp. 1682-1689, 2008.

[42] P. P. Ahern, C. Schiering, S. Buonocore et al., "Interleukin-23 drives intestinal inflammation through direct activity on $\mathrm{T}$ cells," Immunity, vol. 33, no. 2, pp. 279-288, 2010.

[43] R. H. Duerr, K. D. Taylor, S. R. Brant et al., "A genome-wide association study identifies IL23R as an inflammatory bowel disease gene," Science, vol. 314, no. 5804, pp. 1461-1463, 2006.

[44] D. Sivanesan, C. Beauchamp, C. Quinou et al., "IL23R (interleukin 23 receptor) variants protective against inflammatory bowel diseases (IBD) display loss of function due to impaired protein stability and intracellular trafficking," The Journal of Biological Chemistry, vol. 291, no. 16, pp. 8673-8685, 2016.

[45] P. J. Mannon, I. J. Fuss, L. Mayer et al., “Anti-interleukin-12 antibody for active Crohn's disease," The New England Journal of Medicine, vol. 351, no. 20, pp. 2069-2079, 2004.

[46] W. J. Sandborn, B. G. Feagan, R. N. Fedorak et al., "A randomized trial of ustekinumab, a human interleukin-12/ 23 monoclonal antibody, in patients with moderate-tosevere Crohn's disease," Gastroenterology, vol. 135, no. 4, pp. 1130-1141, 2008.

[47] R. Panaccione, W. J. Sandborn, G. L. Gordon et al., "Briakinumab for treatment of Crohn's disease: results of a randomized trial," Inflammatory Bowel Diseases, vol. 21, no. 6, pp. 13291340, 2015.

[48] W. Sandborn, C. Gasink, M. Blank et al., "O-001 a multicenter, double-blind, placebo-controlled phase 3 study of ustekinumab, a human IL-12/23P40 mAB, in moderate-service Crohn's disease refractory to anti-TFN alpha: UNITI-1," 
Inflammatory Bowel Diseases, vol. 22, Supplementary 1, p. S1, 2016.

[49] Q. Guan, Y. Ma, C. L. Hillman et al., "Development of recombinant vaccines against IL-12/IL-23 p40 and in vivo evaluation of their effects in the downregulation of intestinal inflammation in murine colitis," Vaccine, vol. 27, no. 50, pp. 7096-7104, 2009.

[50] Q. Guan, Y. Ma, C. L. Hillman et al., "Targeting IL-12/IL-23 by employing a p40 peptide-based vaccine ameliorates TNBS-induced acute and chronic murine colitis," Molecular Medicine, vol. 17, no. 7-8, pp. 646-656, 2011.

[51] Q. Guan, Y. Ma, L. Aboud et al., “Targeting IL-23 by employing a 40 peptide-based vaccine ameliorates murine allergic skin and airway inflammation," Clinical and Experimental Allergy, vol. 42, no. 9, pp. 1397-1405, 2012.

[52] H. Yoshida and C. A. Hunter, "The immunobiology of interleukin-27," Annual Review of Immunology, vol. 33, pp. 417-443, 2015.

[53] R. R. Meka, S. H. Venkatesha, S. Dudics, B. Acharya, and K. D. Moudgil, "IL-27-induced modulation of autoimmunity and its therapeutic potential," Autoimmunity Reviews, vol. 14, no. 12, pp. 1131-1141, 2015.

[54] C. Andrews, M. H. McLean, and S. K. Durum, "Interleukin27 as a novel therapy for inflammatory bowel disease: a critical review of the literature," Inflammatory Bowel Diseases, vol. 22, no. 9, pp. 2255-2264, 2016.

[55] J. Furuzawa Carballeda, G. Fonseca Camarillo, and J. K. Yamamoto-Furusho, "Interleukin 27 is up-regulated in patients with active inflammatory bowel disease," Immunologic Research, vol. 64, no. 4, pp. 901-907, 2016.

[56] M. L. Hanson, J. A. Hixon, W. Li et al., "Oral delivery of IL-27 recombinant bacteria attenuates immune colitis in mice," Gastroenterology, vol. 146, no. 1, pp. 210-221, 2014, e13.

[57] T. Sasaoka, M. Ito, J. Yamashita et al., "Treatment with IL-27 attenuates experimental colitis through the suppression of the development of IL-17-producing T helper cells," American Journal of Physiology. Gastrointestinal and Liver Physiology, vol. 300, no. 4, pp. G568-G576, 2011.

[58] A. E. Troy, C. Zaph, Y. Du et al., "IL-27 regulates homeostasis of the intestinal CD4+ effector T cell pool and limits intestinal inflammation in a murine model of colitis," Journal of Immunology, vol. 183, no. 3, pp. 2037-2044, 2009.

[59] J. H. Cox, N. M. Kljavin, N. Ramamoorthi, L. Diehl, M. Batten, and N. Ghilardi, "IL-27 promotes T cell-dependent colitis through multiple mechanisms," The Journal of Experimental Medicine, vol. 208, no. 1, pp. 115-123, 2011.

[60] A. Visperas, J. S. Do, K. Bulek, X. Li, and B. Min, "IL-27, targeting antigen-presenting cells, promotes Th17 differentiation and colitis in mice," Mucosal Immunology, vol. 7, no. 3, pp. 625-633, 2014.

[61] C. E. Egwuagu, C. R. Yu, L. Sun, and R. Wang, "Interleukin 35: critical regulator of immunity and lymphocyte-mediated diseases," Cytokine \& Growth Factor Reviews, vol. 26, no. 5, pp. 587-593, 2015.

[62] S. Y. Guan, R. X. Leng, M. I. Khan et al., "Interleukin-35: a potential therapeutic agent for autoimmune diseases," Inflammation, vol. 40, no. 1, pp. 303-310, 2017.

[63] G. Fonseca-Camarillo, J. Furuzawa-Carballeda, and J. K. Yamamoto-Furusho, "Interleukin 35 (IL-35) and IL-37: intestinal and peripheral expression by $\mathrm{T}$ and $\mathrm{B}$ regulatory cells in patients with inflammatory bowel disease," Cytokine, vol. 75, no. 2, pp. 389-402, 2015.

[64] Y. Li, Y. Wang, Y. Liu, X. Zuo, and X. Lu, “The possible role of the novel cytokines il-35 and il-37 in inflammatory bowel disease," Mediators of Inflammation, vol. 2014, Article ID 136329, p. 10, 2014.

[65] S. Wirtz, U. Billmeier, T. McHedlidze, R. S. Blumberg, and M. F. Neurath, "Interleukin-35 mediates mucosal immune responses that protect against T-cell-dependent colitis," Gastroenterology, vol. 141, no. 5, pp. 1875-1886, 2011.

[66] C. Garlanda, C. A. Dinarello, and A. Mantovani, "The interleukin-1 family: back to the future," Immunity, vol. 39, no. 6, pp. 1003-1018, 2013.

[67] J. Palomo, D. Dietrich, P. Martin, G. Palmer, and C. Gabay, "The interleukin (IL)-1 cytokine family-balance between agonists and antagonists in inflammatory diseases," Cytokine, vol. 76, no. 1, pp. 25-37, 2015.

[68] C. Hardman and G. Ogg, "Interleukin-33, friend and foe in type-2 immune responses," Current Opinion in Immunology, vol. 42, pp. 16-24, 2016.

[69] L. Pastorelli, C. De Salvo, M. Vecchi, and T. T. Pizarro, "The role of IL-33 in gut mucosal inflammation," Mediators of Inflammation, vol. 2013, Article ID 608187, p. 11, 2013.

[70] M. S. Islam, K. Horiguchi, S. Iino et al., "Epidermal growth factor is a critical regulator of the cytokine IL-33 in intestinal epithelial cells," British Journal of Pharmacology, vol. 173, no. 16, pp. 2532-2542, 2016.

[71] C. Schiering, T. Krausgruber, A. Chomka et al., "The alarmin IL-33 promotes regulatory T-cell function in the intestine," Nature, vol. 513, no. 7519, pp. 564-568, 2014.

[72] A. Latiano, O. Palmieri, L. Pastorelli et al., "Associations between genetic polymorphisms in IL-33, IL1R1 and risk for inflammatory bowel disease," PloS One, vol. 8, no. 4 article e62144, 2013.

[73] C. J. Beltran, L. E. Nunez, D. Diaz-Jimenez et al., "Characterization of the novel ST2/IL-33 system in patients with inflammatory bowel disease," Inflammatory Bowel Diseases, vol. 16, no. 7, pp. 1097-1107, 2010.

[74] A. Kobori, Y. Yagi, H. Imaeda et al., "Interleukin-33 expression is specifically enhanced in inflamed mucosa of ulcerative colitis," Journal of Gastroenterology, vol. 45, no. 10, pp. 999-1007, 2010.

[75] M. D. Gundersen, R. Goll, J. Hol et al., "Loss of interleukin 33 expression in colonic crypts - a potential marker for disease remission in ulcerative colitis," Scientific Reports, vol. 6, p. 35403, 2016.

[76] D. Diaz-Jimenez, M. De la Fuente, K. Dubois-Camacho et al., "Soluble ST2 is a sensitive clinical marker of ulcerative colitis evolution," BMC Gastroenterology, vol. 16, no. 1, p. 103, 2016.

[77] L. Duan, J. Chen, H. Zhang et al., "Interleukin-33 ameliorates experimental colitis through promoting Th2/Foxp3(+) regulatory T-cell responses in mice," Molecular Medicine, vol. 18, no. 5, pp. 753-761, 2012.

[78] L. A. Monticelli, L. C. Osborne, M. Noti, S. V. Tran, D. M. Zaiss, and D. Artis, "IL-33 promotes an innate immune pathway of intestinal tissue protection dependent on amphiregulin-EGFR interactions," Proceedings of the National Academy of Sciences of the United States of America, vol. 112, no. 34, pp. 10762-10767, 2015.

[79] M.A. Sedhom, M. Pichery, J. R. Murdoch et al., "Neutralisation of the interleukin-33/ST2 pathway ameliorates experimental 
colitis through enhancement of mucosal healing in mice," Gut, vol. 62, no. 12, pp. 1714-1723, 2013.

[80] J. Zhu, F. Yang, L. Sang et al., "IL-33 aggravates DSS-induced acute colitis in mouse colon lamina propria by enhancing Th2 cell responses," Mediators of Inflammation, vol. 2015, Article ID 913041, p. 12, 2015.

[81] P. N. Pushparaj, D. Li, M. Komai-Koma et al., "Interleukin-33 exacerbates acute colitis via interleukin-4 in mice," Immunology, vol. 140, no. 1, pp. 70-77, 2013.

[82] J. Zhu, Y. Wang, F. Yang et al., "IL-33 alleviates DSS-induced chronic colitis in C57BL/6 mice colon lamina propria by suppressing Th17 cell response as well as Th1 cell response," International Immunopharmacology, vol. 29, no. 2, pp. 846853, 2015.

[83] C. De Salvo, X. M. Wang, L. Pastorelli et al., "IL-33 drives eosinophil infiltration and pathogenic type 2 helper T-cell immune responses leading to chronic experimental ileitis," The American Journal of Pathology, vol. 186, no. 4, pp. 885898, 2016.

[84] G. Bamias and F. Cominelli, "Cytokines and intestinal inflammation," Current Opinion in Gastroenterology, vol. 32, no. 6, pp. 437-442, 2016.

[85] M. S. Gresnigt and F. L. van de Veerdonk, "Biology of IL-36 cytokines and their role in disease," Seminars in Immunology, vol. 25, no. 6, pp. 458-465, 2013.

[86] Y. Carrier, H. L. Ma, H. E. Ramon et al., "Inter-regulation of Th17 cytokines and the IL-36 cytokines in vitro and in vivo: implications in psoriasis pathogenesis," The Journal of Investigative Dermatology, vol. 131, no. 12, pp. 2428-2437, 2011.

[87] S. Vigne, G. Palmer, C. Lamacchia et al., "IL-36R ligands are potent regulators of dendritic and T cells," Blood, vol. 118, no. 22, pp. 5813-5823, 2011.

[88] S. Mutamba, A. Allison, Y. Mahida, P. Barrow, and N. Foster, "Expression of IL-1Rrp2 by human myelomonocytic cells is unique to DCs and facilitates DC maturation by IL-1F8 and IL-1F9," European Journal of Immunology, vol. 42, no. 3, pp. 607-617, 2012.

[89] A. Nishida, K. Hidaka, T. Kanda et al., "Increased expression of interleukin-36, a member of the interleukin-1 cytokine family, in inflammatory bowel disease," Inflammatory Bowel Diseases, vol. 22, no. 2, pp. 303-314, 2016.

[90] M. A. Boutet, G. Bart, M. Penhoat et al., "Distinct expression of interleukin (IL)-36alpha, beta and gamma, their antagonist IL-36Ra and IL-38 in psoriasis, rheumatoid arthritis and Crohn's disease," Clinical and Experimental Immunology, vol. 184, no. 2, pp. 159-173, 2016.

[91] S. E. Russell, R. M. Horan, A. M. Stefanska et al., "IL-36alpha expression is elevated in ulcerative colitis and promotes colonic inflammation," Mucosal Immunology, vol. 9, no. 5, pp. 1193-1204, 2016.

[92] O. Medina-Contreras, A. Harusato, H. Nishio et al., "Cutting edge: IL-36 receptor promotes resolution of intestinal damage," Journal of Immunology, vol. 196, no. 1, pp. 34-38, 2016.

[93] W. D. Xu, Y. Zhao, and Y. Liu, "Insights into IL-37, the role in autoimmune diseases," Autoimmunity Reviews, vol. 14, no. 12, pp. 1170-1175, 2015.

[94] C. A. Dinarello, C. Nold-Petry, M. Nold et al., "Suppression of innate inflammation and immunity by interleukin-37," European Journal of Immunology, vol. 46, no. 5, pp. 10671081, 2016.
[95] C. A. Dinarello and P. Bufler, "Interleukin-37," Seminars in Immunology, vol. 25, no. 6, pp. 466-468, 2013.

[96] P. Bufler, F. Gamboni-Robertson, T. Azam, S. H. Kim, and C. A. Dinarello, "Interleukin-1 homologues IL-1F7b and IL-18 contain functional mRNA instability elements within the coding region responsive to lipopolysaccharide," The Biochemical Journal, vol. 381, no. Pt 2, pp. 503-510, 2004.

[97] S. Weidlich, A. M. Bulau, T. Schwerd et al., "Intestinal expression of the anti-inflammatory interleukin-1 homologue IL-37 in pediatric inflammatory bowel disease," Journal of Pediatric Gastroenterology and Nutrition, vol. 59, no. 2, pp. e18-e26, 2014.

[98] H. Imaeda, K. Takahashi, T. Fujimoto et al., "Epithelial expression of interleukin-37b in inflammatory bowel disease," Clinical and Experimental Immunology, vol. 172, no. 3, pp. 410-416, 2013.

[99] E. N. McNamee, J. C. Masterson, P. Jedlicka et al., "Interleukin 37 expression protects mice from colitis," Proceedings of the National Academy of Sciences of the United States of America, vol. 108, no. 40, pp. 16711-16716, 2011.

[100] W. Q. Wang, K. Dong, L. Zhou et al., "IL-37b gene transfer enhances the therapeutic efficacy of mesenchumal stromal cells in DSS-induced colitis mice," Acta Pharmacologica Sinica, vol. 36, no. 11, pp. 1377-1387, 2015. 


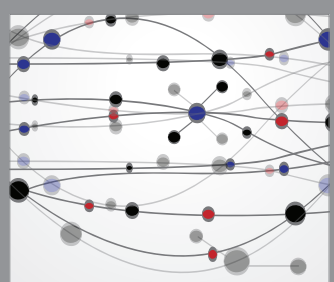

The Scientific World Journal
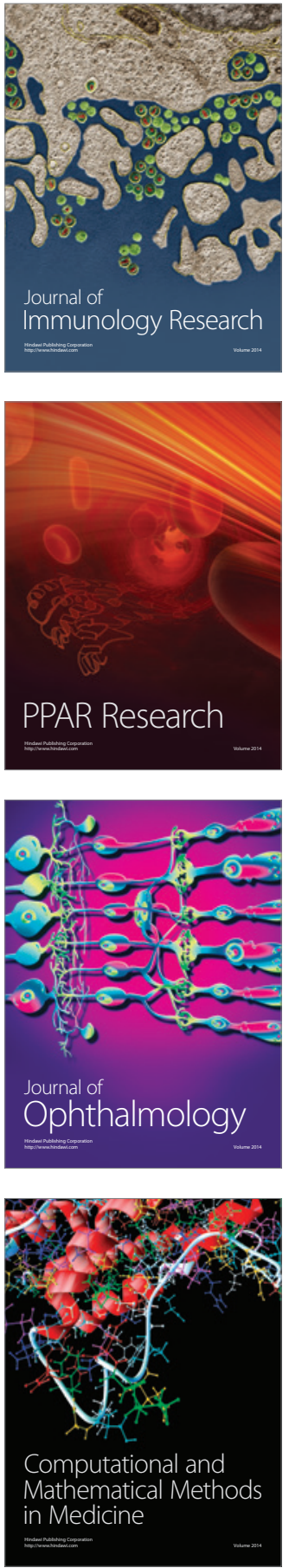

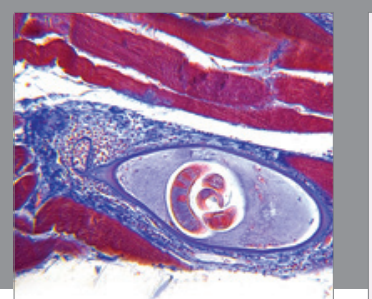

Gastroenterology Research and Practice
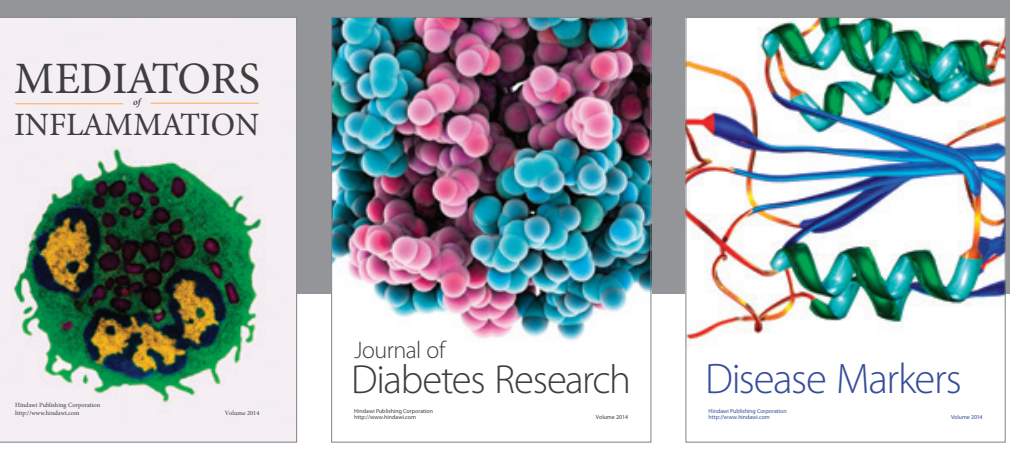

Disease Markers

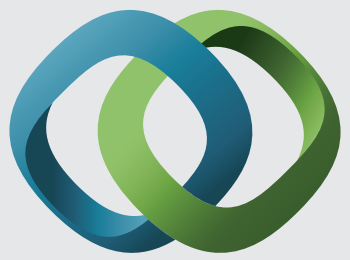

\section{Hindawi}

Submit your manuscripts at

https://www.hindawi.com
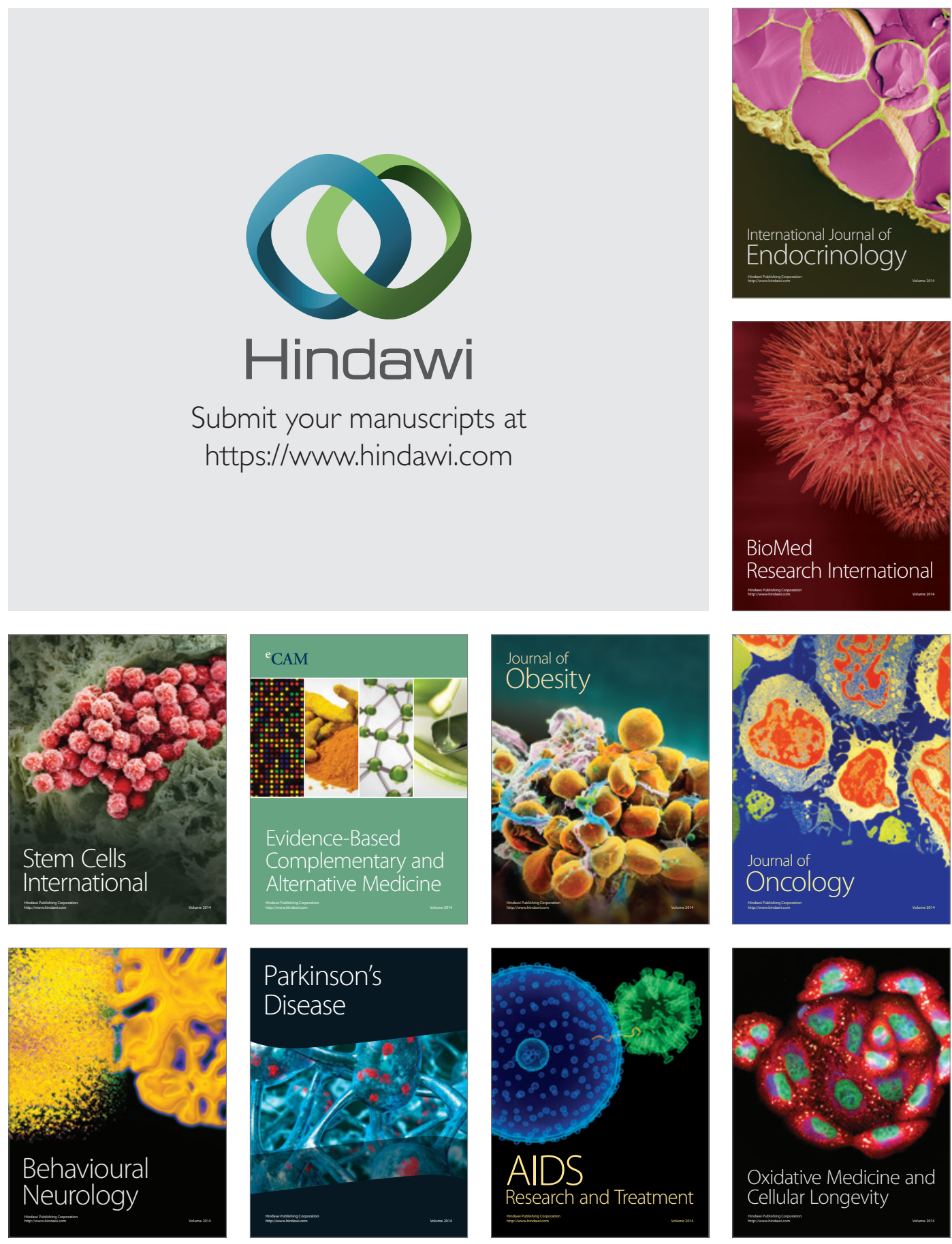